Annals of Mathematics, 149 (1999), 511-533

\title{
Local inequalities for plurisubharmonic functions
}

\author{
By Alexander Brudnyi*
}

\begin{abstract}
The main objective of this paper is to prove a new inequality for plurisubharmonic functions estimating their supremum over a ball by their supremum over a measurable subset of the ball. We apply this result to study local properties of polynomial, algebraic and analytic functions. The paper has much in common with an earlier paper $[\mathrm{Br}]$ of the author.
\end{abstract}

\section{Introduction and formulation of main results}

1. A real-valued function $f$ defined on a domain $\Omega \subset \mathbb{C}^{n}$ is called plurisubharmonic in $\Omega$ if $f$ is upper semicontinuous and its restriction to components of a complex line intersected with $\Omega$ is subharmonic.

The main objective of this paper is to prove a new inequality for plurisubharmonic functions estimating their supremum over a ball by supremum over a measurable subset of the ball. The inequality has many applications, several of which are presented in this paper. To formulate the result and its applications we introduce

Definition 1.1. A plurisubharmonic function $f: \mathbb{C}^{n} \longrightarrow \mathbb{R}$ belongs to class $\mathcal{F}_{r}(r>1)$ if it satisfies

$$
\begin{aligned}
& \sup _{B_{c}(0, r)} f=0 \\
& \sup _{B_{c}(0,1)} f \geq-1 .
\end{aligned}
$$

Hereafter $B(x, \rho)$ and $B_{c}(x, \rho)$ denote the Euclidean ball with center $x$ and radius $\rho$ in $\mathbb{R}^{n}$ and $\mathbb{C}^{n}$, respectively.

\footnotetext{
${ }^{*}$ Research supported in part by NSERC. 1991 Mathematics Subject Classification. Primary 31B05. Secondary 46 E15.

Key words and phrases. Yu. Brudnyǐ-Ganzburg type inequality, plurisubharmonic function, BMOfunction.
} 
Let the ball $B(x, t)$ satisfy

$$
B(x, t) \subset B_{c}(x, a t) \subset B_{c}(0,1),
$$

where $a>1$ is a fixed constant.

THEOREM 1.2. There are constants $c=c(a, r)$ and $d=d(n)^{1}$ such that the inequality

$$
\sup _{B(x, t)} f \leq c \log \left(\frac{d|B(x, t)|}{|\omega|}\right)+\sup _{\omega} f
$$

holds for every $f \in \mathcal{F}_{r}$ and every measurable subset $\omega \subset B(x, t)$.

To illustrate the possible applications of the main result, let us consider a real polynomial $p \in \mathbb{R}\left[x_{1}, \ldots, x_{n}\right]$ of degree at most $k$ (we will denote the space of these polynomials by $\left.\mathcal{P}_{k, n}(\mathbb{R})\right)$. According to the classical Bernstein "doubling" inequality

$$
\max _{B_{c}(0, r)}|p| \leq r^{k} \max _{B_{c}(0,1)}|p| \quad(r>1) .
$$

Consider the plurisubharmonic function

$$
F_{r}(z):=(\log r)^{-1} k^{-1}\left(\log |p(z)|-\sup _{B_{c}(0, r)} \log |p|\right) \quad\left(z \in \mathbb{C}^{n}\right) .
$$

From the definition of $\mathcal{F}_{r}$ and (1.3) it follows that $F_{r} \in \mathcal{F}_{r}$ for any $r>1$. Applying (1.2) with $r=2$ to this function we get

$$
\sup _{B}|p| \leq\left(\frac{d|B|}{|\omega|}\right)^{c k} \sup _{\omega}|p|
$$

for an arbitrary ball $B$ and its measurable subset $\omega$.

In fact, in this case, we can take $c=1$ and $d=4 n$ as follows from the sharp inequality due to Remez [R] for $n=1$ and Yu. Brudnyí-Ganzburg [BG] 2 in the general case.

2. Applications of the main theorem are related to Yu. Brudnyĩ-Ganzburg type inequalities for polynomials, algebraic functions and entire functions of exponential type. We give also applications to log-BMO properties of real analytic functions, which previously were known only for polynomials (see [St]). As is seen from the proof of (1.4) the main result serves in these applications as a kind of amplifier, transforming weak-type inequalities into strong-type

\footnotetext{
${ }^{1}$ Here and below the notation $C=C(\alpha, \beta, \gamma, \ldots)$ means that the constant depends only on the parameters $\alpha, \beta, \gamma, \ldots$.

${ }^{2}$ In the original version the ratio on the right-hand side of (1.4) can be replaced by $T_{k}\left(\frac{1+\beta_{n}(\lambda)}{1-\beta_{n}(\lambda)}\right)$ with $\lambda:=\frac{|\omega|}{|B|}$ and $\beta_{n}(\lambda)=(1-\lambda)^{\frac{1}{n}}$. Here $T_{k}$ is the Tchebychef polynomial of degree $k$.
} 
ones. Of course, these "weak" inequalities are, clearly, highly nontrivial and obtaining them may require a great deal of effort. Fortunately, a number of these have recently been proved in connection with different aspects of modern analysis (see, in particular, [S], [FN1], [FN2], [FN3], [Br], [BMLT], [RY], [LL]).

3. We now formulate two consequences of the main result which give a refinement (and a relatively simple alternative proof) of the basic results of $[\mathrm{Br}]$ and $[\mathrm{FN} 3]$. We begin with a sharpening of the main result in $[\mathrm{Br}]$ (in the original version of inequality (1.5) below the exponent depends on $k$ in a nonlinear way).

To formulate the result suppose that $V \subset \mathbb{R}^{n}$ is a real algebraic variety of pure dimension $m(1 \leq m \leq n-1)$. We endow $V$ with the metric and the measure induced from the Euclidean metric and Lebesgue measure of $\mathbb{R}^{n}$.

TheOREM 1.3. For every regular point $x \in V$ there is an open neighborhood $N=N(V)$ of $x$ such that

$$
\sup _{B}|p| \leq\left(\frac{d \lambda_{V}(B)}{\lambda_{V}(\omega)}\right)^{\alpha k \operatorname{deg}(V)} \sup _{\omega}|p|
$$

for every ball $B \subset N$, measurable subset $\omega \subset B$ and polynomial $p \in \mathcal{P}_{k, n}(\mathbb{R})$.

Here $\lambda_{V}$ denotes the induced Lebesgue measure in $V$ and $d=d(m)$ and $\alpha$ is an absolute constant.

Our next result is a generalization of the first main result in [FN3] in which $\omega$ in (1.6) below is a ball.

THEOREM 1.4. Let $F_{1, \lambda}, \ldots, F_{N, \lambda}$ be holomorphic functions on the ball $B_{c}\left(0,1+r_{0}\right) \subset \mathbb{C}^{n}, r_{0}>0$, depending real-analytically on $\lambda \in U \subset \mathbb{R}^{m}$ where $U$ is open. Let $V_{\lambda}$ be the linear span of the $F_{k, \lambda}, 1 \leq k \leq N$. Then for any compact set $K \subset U$, there is a constant $\gamma=\gamma\left(K, r_{0}\right)>0$ such that the $Y u$. Brudnyĭ-Ganzburg type inequality

$$
\sup _{B(x, \rho)}|F| \leq\left(\frac{d(n)|B(x, \rho)|}{|\omega|}\right)^{\gamma} \sup _{\omega}|F|
$$

holds for any $F \in V_{\lambda}, \lambda \in K$ and $\omega \subset B(x, \rho) \subset B(0,1)$.

4. Our next results deal with log-BMO properties of algebraic and analytic functions. The estimate of Theorem 1.2 implies BMO-norm estimates for important classes of analytic functions. We formulate only a few results of this kind. Our first result completes Theorem 5.5 of $[\mathrm{Br}]$.

TheOREM 1.5. Let $V$ be a compact algebraic submanifold of $\mathbb{R}^{n}$. Then for every real polynomial $p \in \mathcal{P}_{k, n}(\mathbb{R})$ with $\left.p\right|_{V} \neq 0$ the function $\left.(\log |p|)\right|_{V} \in$ $\mathrm{BMO}(V)$ and its $\mathrm{BMO}-n o r m$ is bounded above by $C(V) k$. 
Let us recall that the BMO-norm of $f \in L_{1}\left(V, d \lambda_{V}\right)$ is defined as

$$
|f|_{*}:=\sup _{B} \frac{1}{\lambda_{V}(B)} \int_{B}\left|f-f_{B}\right| d \lambda_{V}
$$

where $f_{B}:=\frac{1}{\lambda_{V}(B)} \int_{B} f d \lambda_{V}, B \subset V$ is a ball with respect to the induced metric and $\lambda_{V}$ is the Lebesgue measure on $V$ induced from $\mathbb{R}^{n}$.

Remark 1.6. In the previous version of this result, the BMO-norm was estimated by a constant depending nonlinearly on the degree $k$.

Now let $\left\{F_{j, \lambda}\right\}_{1 \leq j \leq N}$ be a family of real analytic functions defined on a compact real analytic manifold $V$ and depending real-analytically on $\lambda$ varying in an open subset $U$ of $\mathbb{R}^{m}$.

Theorem 1.7. Let $V_{\lambda}:=\operatorname{span}\left\{F_{j, \lambda}\right\}_{1 \leq j \leq N}$. Then for every compact set $K \subset U$ there is a constant $C=C(K)>0$ such that

$$
|\log | F||_{*} \leq C
$$

for every $F \in V_{\lambda}$ with $\lambda \in K$.

\section{Proof of Theorem 1.2}

1. The proof is divided into three parts, the first of which will be presented in this section. It contains several auxiliary results on subharmonic and plurisubharmonic functions.

Let $\mathcal{P S H}(\Omega)$ denote the class of plurisubharmonic in $\Omega$ functions. An important subclass of $\mathcal{P} \mathcal{S H}\left(\mathbb{C}^{n}\right)$ is introduced as follows.

Definition 2.1. A function $u \in \mathcal{P S H}\left(\mathbb{C}^{n}\right)$ belongs to the class $\mathcal{L}\left(\mathbb{C}^{n}\right)$ (of functions of minimal growth) if

$$
u(z)-\log (1+|z|) \leq \alpha \quad\left(z \in \mathbb{C}^{n}\right)
$$

for a constant $\alpha$.

To formulate our first auxiliary result consider the family $\mathcal{A}_{r}$ of continuous nonpositive subharmonic functions $f: \mathbb{D} \longrightarrow \mathbb{R}$ such that

$$
-1 \leq \sup _{\mathbb{D}_{r}} f
$$

Here $\mathbb{D}_{r}:=\{z \in \mathbb{C} ;|z|<r\}, \mathbb{D}:=\mathbb{D}_{1}$ and $r$ is a fixed number, $0<r<1$.

Proposition 2.2. For every $f \in \mathcal{A}_{r}$ there exists a subharmonic function $h_{f}: \mathbb{C} \longrightarrow \mathbb{R}$ and a constant $c_{f}>0$ such that 


$$
\begin{gathered}
h_{f} / c_{f} \in \mathcal{L}(\mathbb{C}) ; \\
f=h_{f} \text { on } \mathbb{D}_{r} ; \\
\sup _{f \in \mathcal{A}_{r}} c_{f}<\infty .
\end{gathered}
$$

Proof. Let $R:=\left\{\frac{1+3 r}{4} \leq|z| \leq \frac{1+r}{2}\right\}$ be an annulus in $\mathbb{D} \backslash \mathbb{D}_{r}$, and let $X$ denote the family of concentric circles centered at 0 and contained in $R$.

Lemma 2.3. Let $f \in \mathcal{A}_{r}$ and

$$
t(f):=\sup _{S \in X} \inf _{z \in S} f(z) .
$$

Then

$$
C(r):=\inf _{f \in \mathcal{A}_{r}} t(f)>-\infty
$$

Proof. Below we follow a scheme suggested by N. Levenberg that essentially simplifies our original proof. Let $\left\{f_{i}\right\}_{i \geq 1} \subset \mathcal{A}_{r}$ be such that

$$
\lim _{i \rightarrow \infty} t\left(f_{i}\right)=C(r) .
$$

Without loss of generality we may assume that the sequence does not contain the zero function. For every $S \subset X$ we set

$$
S_{i}:=\left\{z \in S ; f_{i}(z)=\min _{S} f_{i}\right\}
$$

and

$$
K_{i}:=\bigcup_{S \in X} S_{i}
$$

By the continuity of $f_{i}$ the set $K_{i}$ is compact. The set of radii of points in $K_{i}$ fills out the interval $I_{r}:=\left[\frac{1+3 r}{4}, \frac{1+r}{2}\right]$ of length $w(r):=\frac{1-r}{4}$. Then the transfinite diameter $\delta\left(K_{i}\right)$ of $K_{i}$ satisfies

$$
\delta\left(K_{i}\right) \geq \delta\left(I_{r}\right)=\frac{w(r)}{4} .
$$

(See [G, Chap. VII], for the definition and properties of transfinite diameter.) Now we set

$$
m_{i}:=\max _{K_{i}} f_{i} \quad \text { and } \quad g_{i}:=\frac{f_{i}}{\left|m_{i}\right|} .
$$

Here $m_{i}<0$, for otherwise $f_{i}$ equals 0 identically. To complete the proof we must estimate $\left|m_{i}\right|$ by a constant independent of $i \geq 1$. To this end we will compare $g_{i}$ with the relative extremal function $u_{K_{i}, \mathbb{D}}$ of the pair $\left(K_{i}, \mathbb{D}\right)$. Recall that the latter is defined by

$$
u_{K_{i}, \mathbb{D}}(z):=\sup \left\{v(z): \quad v \in \mathcal{S H}(\mathbb{D}),\left.v\right|_{K_{i}} \leq-1, v \leq 0\right\}
$$


for $z \in \mathbb{D}$. Here $\mathcal{S H}(\mathbb{D})=\mathcal{P} \mathcal{S H}(\mathbb{D})$ for $n=1$. Since $g_{i} \leq-1$ on $K_{i}$ by definition, we have

$$
g_{i} \leq u_{K_{i}, \mathbb{D}} .
$$

Let now $\left(u_{K_{i}, \mathbb{D}}\right)^{*}$ be the upper semicontinuous regularization of $(2.6)$. Then this function is subharmonic in $\mathbb{D}$, see, e.g. $[\mathrm{K}]$. By the nonpositivity of both the regularization and $g_{i}$ and by inequality $(2.7)$ we have

$$
\left|\left(u_{K_{i}, \mathbb{D}}\right)^{*}\right| \leq\left|g_{i}\right|=\frac{\left|f_{i}\right|}{\left|m_{i}\right|}
$$

as well. From here it follows that at a certain point $z_{0} \in \mathbb{D}$, which we will specify later, we get

$$
\left|m_{i}\right| \leq \frac{\left|f_{i}\left(z_{0}\right)\right|}{\left|\left(u_{K_{i}, \mathbb{D}}\right)^{*}\left(z_{0}\right)\right|}
$$

To select $z_{0}$ and to estimate the denominator in (2.8) we make use of the relation between the relative extremal function and the capacity $\operatorname{cap}\left(K_{i}, \mathbb{D}\right)$ which is defined by

$$
\operatorname{cap}\left(K_{i}, \mathbb{D}\right):=\int_{\mathbb{D}} \Delta\left(u_{K_{i}, \mathbb{D}}\right)^{*} d x d y
$$

see, e.g., $[\mathrm{K}]$. Since $\left(u_{K_{i}, \mathbb{D}}\right)^{*}$ satisfies the Laplace equation outside of $K_{i}$ we can rewrite the right side as follows.

Let $R^{\prime} \subset \mathbb{D}$ be an arbitrary annulus outside of the $\operatorname{circle} \operatorname{conv}(R)=$ $\left\{z ;|z| \leq \frac{1+r}{2}\right\}$ and $\rho$ be a smooth function with support in $\operatorname{conv}\left(R^{\prime}\right)$ that equals 1 in $\operatorname{conv}\left(R^{\prime}\right) \backslash R^{\prime}$. Then by Green's formula

$$
\operatorname{cap}\left(K_{i}, \mathbb{D}\right)=\int_{\mathbb{D}} \rho \Delta\left(u_{K_{i}, \mathbb{D}}\right)^{*} d x d y=\left|\int_{R^{\prime}}\left(u_{K_{i}, \mathbb{D}}\right)^{*} \Delta \rho d x d y\right| \leq C \max _{R^{\prime}}\left|\left(u_{K_{i}, \mathbb{D}}\right)^{*}\right| .
$$

Since the function $\left(u_{K_{i}, \mathbb{D}}\right)^{*}$ is nonpositive and harmonic in $\mathbb{D} \backslash K_{i}$, Harnack's inequality (see, e.g., [K, Lemma 2.2.9]) implies

$$
\max _{R^{\prime}}\left|\left(u_{K_{i}, \mathbb{D}}\right)^{*}\right| \leq C^{\prime}\left|\left(u_{K_{i}, \mathbb{D}}\right)^{*}\left(z_{0}\right)\right|
$$

for a constant $C^{\prime}$ (depending on $r$ only) and every $z_{0} \in R^{\prime}$.

Putting together (2.8) and the latter two inequalities we find that the inequality

$$
\left|m_{i}\right| \leq \frac{C^{\prime \prime}\left|f_{i}\left(z_{0}\right)\right|}{\operatorname{cap}\left(K_{i}, \mathbb{D}\right)}
$$

holds for every $z_{0} \in R^{\prime}$. But by the definition of $\mathcal{A}_{r}$ and the maximum principle, $0>\max _{R^{\prime}} f_{i} \geq-1$. Taking $z_{0}$ as a point at which the latter maximum is attained, we then get

$$
\left|m_{i}\right| \leq \frac{C^{\prime \prime}}{\operatorname{cap}\left(K_{i}, \mathbb{D}\right)}
$$


It remains to apply the one-dimensional version of the comparison theorem of Alexander and Taylor, see [AT], that gives the following inequality for the transfinite diameter of $K_{i}$ (which coincides with the logarithmic capacity of $K_{i}$ ):

$$
\delta\left(K_{i}\right) \leq \exp \left(-\frac{2 \pi}{\operatorname{cap}\left(K_{i}, \mathbb{D}\right)}\right) .
$$

Putting together the latter two inequalities and inequality (2.4) we finally obtain

$$
\left|m_{i}\right| \leq C^{\prime \prime \prime} \log \left(\frac{4}{w(r)}\right)=: C^{\prime \prime \prime}(r)
$$

for every $i \geq 1$. By the definition of the $\left\{f_{i}\right\}$ it follows that

$$
\inf _{f \in \mathcal{A}_{r}} t(f)=\lim _{i \rightarrow \infty} t\left(f_{i}\right) \geq-\inf _{i}\left|m_{i}\right| \geq-C^{\prime \prime \prime}(r)>-\infty .
$$

The lemma is proved.

Now we are in a position to prove Proposition 2.2. Let $f \in \mathcal{A}_{r}$. According to the lemma there exists a circle $S_{f} \in X$ such that

$$
\inf _{S_{f}} f \geq C(r)>-\infty .
$$

$S_{f}$ is the boundary of the disk $\mathbb{D}_{r(f)}$, where

$$
\frac{1+3 r}{4} \leq r(f) \leq \frac{1+r}{2} \text {. }
$$

We now define the required subharmonic function $h_{f}(z): \mathbb{C} \longrightarrow \mathbb{R}$ by

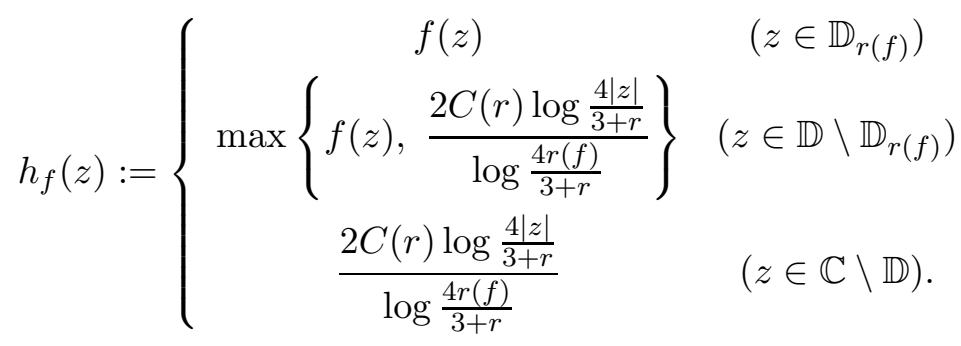

Since the ratio in the third formula is less than $C(r)<0$ on $S_{f}$ and greater than 0 on $\partial \mathbb{D}$, and since $f$ is continuous, $h_{f}$ is subharmonic on $\mathbb{C}$. Moreover, according to Definition 2.1,

$$
\frac{\log \frac{4 r(f)}{3+r}}{2 C(r)} h_{f} \in \mathcal{L}(\mathbb{C}) .
$$

It remains to define

$$
c_{f}:=\frac{\log \frac{4 r(f)}{3+r}}{2 C(r)} .
$$

Then

$$
c_{f} \leq \frac{\log \frac{1+3 r}{3+r}}{2 C(r)}<\infty
$$

and the proposition is proved. 
The final result of this section discusses an approximation theorem for plurisubharmonic functions which will allow us to reduce the proof to the case of $C^{\infty}$-functions.

Let $\kappa$ be a nonnegative radial $C^{\infty}$-function on $\mathbb{C}^{n}$ satisfying

$$
\int_{\mathbb{C}^{n}} \kappa(x) d x d y=1, \quad \operatorname{supp}(\kappa) \subset B_{c}(0,1),
$$

where $z=x+i y, \quad x, y \in \mathbb{R}^{n}$. Let $\Omega \subset \mathbb{C}^{n}$ be a domain. For $f \in \mathcal{P} \mathcal{S H}(\Omega)$, we let $f_{\varepsilon}$ denote the function defined by

$$
f_{\varepsilon}(w):=\int_{\mathbb{C}^{n}} \kappa(z) f(w-\varepsilon z) d x d y,
$$

where $w \in \Omega_{\varepsilon}:=\{z \in \Omega$ : $\operatorname{dist}(z, \partial \Omega)>\varepsilon\}$. It is well known, see, e.g., [K, Th. 2.9.2], that $f_{\varepsilon} \in C^{\infty} \cap \mathcal{P S H}\left(\Omega_{\varepsilon}\right)$ and that $f_{\varepsilon}(w)$ monotonically decreases and tends to $f(w)$ for each $w \in \Omega$ as $\varepsilon \rightarrow 0$.

Lemma 2.4. Let $f \in \mathcal{F}_{r}$. Assume that the functions $\left\{f_{1 / k}\right\}_{k \geq k_{0}}$ satisfy inequality (1.2) with $B(x, t)$ and a compact $\omega$ independent of $k$. Then $f$ also satisfies this inequality.

Proof. Since $f$ is defined on $B_{c}(0, r)$ with $r>1$, the function $f_{1 / k}$ belongs to $C^{\infty} \cap \mathcal{P} \mathcal{S H}\left(B_{c}(0, r-1 / k)\right)$. Let $\left\{w_{k}\right\}_{k \geq 1} \subset \omega$ be such that

$$
f_{1 / k}\left(w_{k}\right)=\max _{\omega} f_{1 / k}
$$

Moreover, let $z_{\varepsilon, t} \in B(x, t)$ be a point such that

$$
\sup _{B(x, t)} f-f\left(z_{\varepsilon, t}\right)<\varepsilon .
$$

According to the assumptions of the lemma

$$
f\left(z_{\varepsilon, t}\right)=\lim _{k \rightarrow \infty} f_{1 / k}\left(z_{\varepsilon, t}\right) \leq c \log \frac{d|B(x, t)|}{|\omega|}+\limsup _{k \rightarrow \infty} f_{1 / k}\left(w_{k}\right) .
$$

To estimate the second summand let us use (2.9) and (2.10):

$$
f_{1 / k}\left(w_{k}\right)=\int_{\mathbb{C}^{n}} \kappa(z) f\left(w_{k}-z / k\right) d x d y \leq \sup _{B_{c}\left(w_{k}, 1 / k\right)} f .
$$

Now let $x_{k} \in B_{c}\left(w_{k}, 1 / k\right)$ be such that the supremum on the right is less than $f\left(x_{k}\right)+1 / k$. Because of the compactness of $\omega$ we can assume that $w:=$ $\lim _{k \rightarrow \infty} w_{k}$ exists. Then we have $\lim _{k \rightarrow \infty} x_{k}=\lim _{k \rightarrow \infty} w_{k}=w \in \omega$. Using the upper semicontinuity of $f$ it follows that

$$
\limsup _{k \rightarrow \infty} \sup _{B_{c}\left(w_{k}, 1 / k\right)} f \leq \limsup _{k \rightarrow \infty} f\left(x_{k}\right) \leq f(w) \leq \sup _{\omega} f,
$$

which leads to the inequality

$$
\limsup _{k \rightarrow \infty} f_{1 / k}\left(w_{k}\right) \leq \sup _{\omega} f .
$$


Putting this inequality together with (2.11) and (2.12) and letting $\varepsilon \rightarrow 0$, we get

$$
\sup _{B(x, t)} f \leq c \log \frac{d|B(x, t)|}{|\omega|}+\sup _{\omega} f .
$$

The proof is complete.

2. The second part of the proof of Theorem 1.2 is the Bernstein "doubling" inequality for functions in $\mathcal{F}_{r}$.

Proposition 2.5. Let $f \in \mathcal{F}_{r}$ and $s \in[1, a], a>1$. Suppose that

$$
B_{c}(x, t) \subset B_{c}(x, a t) \subset B_{c}(0,1) .
$$

Then there is a constant $c=c(r)$ such that

$$
\sup _{B_{c}(x, s t)} f \leq c \log s+\sup _{B_{c}(x, t)} f .
$$

Proof. Consider the pair of embedded balls $B_{c}\left(x, \frac{r-|x|}{r}\right) \subset B_{c}(x, r-|x|)$, where $x \in B_{c}(0,1)$ and $|\cdot|$ denotes the Euclidean norm. The smaller ball contains $B_{c}(x, 1-|x|)$ which has maximal radius of balls in $B_{c}(x, 1)$ centered at $x$. From (2.14) it follows that

$$
B_{c}(x, a t) \subset B_{c}\left(x, \frac{r-|x|}{r}\right) .
$$

Let

$$
\gamma_{r}(f ; x):=\sup _{B_{c}\left(x, \frac{r-|x|}{r}\right)} f
$$

and

$$
\gamma_{r}:=\inf _{f \in \mathcal{F}_{r}} \inf _{x \in B_{c}(0,1)} \gamma_{r}(f ; x)
$$

Clearly, if $r_{1} \leq r_{2}$ then

$$
\gamma_{r_{1}}(f ; x) \leq \gamma_{r_{2}}(f ; x) \quad \text { and } \quad \gamma_{r_{1}} \leq \gamma_{r_{2}} .
$$

LEMMA 2.6. There is a nonpositive constant $C=C(r)$ such that the inequality

$$
\gamma_{r} \geq \lim _{k \rightarrow \infty} \gamma_{r_{k}} \geq C>-\infty
$$

holds for every $\left\{r_{k}\right\}_{k \geq 1}$ increasing to $r$.

Proof. According to (2.18), $\left\{\gamma_{r_{k}}\right\}_{k \geq 1}$ is a monotone nondecreasing sequence and therefore $\lim _{k \rightarrow \infty} \gamma_{r_{k}}(\in[-\infty, 0])$ does exist. Let $\left\{f_{k} \in \mathcal{F}_{r_{k}}\right\}_{k \geq 1}$ and $\left\{x_{k}\right\}_{k \geq 1} \subset B_{c}(0,1)$ be chosen such that

$$
\lim _{k \rightarrow \infty} \gamma_{r_{k}}\left(f_{k} ; x_{k}\right)=\lim _{k \rightarrow \infty} \gamma_{r_{k}}
$$


Here we may assume that each $f_{k}$ does not identically equal 0 . Let $B_{k}$ denote the ball $B_{c}\left(x_{k}, \frac{r_{k}-\left|x_{k}\right|}{r_{k}}\right)$ and $\lambda B$ denote the homothety of $B$ with center 0 and dilation coefficient $\lambda>0$. Consider the sequence of balls

$$
\left\{t_{k} B_{k}\right\}_{k \geq 1}, \quad \text { where } \quad t_{k}:=\left(r / r_{k}\right)>1
$$

and the sequence of functions

$$
f_{k}^{\prime}(z):=f_{k}\left(z / t_{k}\right) \quad\left(z \in B_{c}(0, r)\right) .
$$

Then we have

$$
\gamma_{r_{k}}\left(f_{k} ; x_{k}\right)=\sup _{t_{k} B_{k}} f_{k}^{\prime}
$$

Without loss of generality we assume that $\left\{t_{k} x_{k}\right\}_{k \geq 1}$ converges to $x \in B_{c}(0,1)$. Then the limit ball $B_{c}\left(x, \frac{r-|x|}{r}\right)$ of the sequence $\left\{t_{k} B_{k}\right\}$ has radius at least $l:=$ $\frac{r-1}{r}$. Therefore its intersection with $B_{c}(0,1)$ contains the ball $B^{l}:=B_{c}(y, l / 4)$, where $y=x\left(1-\frac{r-|x|}{2 r|x|}\right)$. Passing to a subsequence we may assume that

$$
B^{l} \subset t_{k} B_{k}
$$

for all $k \geq 1$. It follows that

$$
m_{k}:=\sup _{B^{l}} f_{k}^{\prime} \leq \gamma_{r_{k}}\left(f_{k} ; x_{k}\right)<0 .
$$

Consider now the sequence $\left\{f_{k}^{\prime \prime}:=f_{k}^{\prime} /\left|m_{k}\right|\right\}_{k \geq 1}$. Each function of the sequence is less than or equal to -1 on $B^{l}$ and nonpositive on $B_{c}(0, r)$. Therefore it is bounded above by the relative extremal function

$$
u_{B^{l}, B_{c}(0, r)}:=\sup \left\{v(z): v \in \mathcal{P S H}\left(B_{c}(0, r)\right),\left.v\right|_{B^{l}} \leq-1, v \leq 0\right\} .
$$

Since the compact ball $B^{l}$ is pluriregular, this function is continuous and strictly negative outside $B^{l}$ (see, e.g., [K, Cor. 4.5.9]). Therefore

$$
M\left(r_{k}\right):=\max _{\partial B_{c}\left(0, t_{k}\right)} u_{B^{l}, B_{c}(0, r)}<0
$$

and

$$
\left|f_{k}^{\prime \prime}(z)\right| \geq\left|M\left(r_{k}\right)\right|
$$

for every $z \in \partial B_{c}\left(0, t_{k}\right)$. From this, the definition of $f_{k}^{\prime \prime}$ and inequality (2.19), it follows that

$$
\left|f_{k}^{\prime}(z)\right| \geq\left|M\left(r_{k}\right) \gamma_{r_{k}}\left(f_{k} ; x_{k}\right)\right| \quad\left(z \in \partial B_{c}\left(0, t_{k}\right)\right) .
$$

But the supremum of $f_{k}^{\prime}$ over $B_{c}\left(0, t_{k}\right)$ is at least -1 . So the previous inequality yields

$$
\left|\gamma_{r_{k}}\left(f_{k} ; x_{k}\right)\right| \leq \frac{1}{\left|M\left(r_{k}\right)\right|}
$$


Letting $k \rightarrow \infty$ we conclude that

$$
\left|\lim _{k \rightarrow \infty} \gamma_{r_{k}}\left(f_{k} ; x_{k}\right)\right| \leq \frac{1}{|M(r)|}
$$

where

$$
M(r):=\max _{\partial B_{c}(0,1)} u_{B^{l}, B_{c}(0, r)}<0 .
$$

The proof of the lemma is complete.

We now proceed to prove Proposition 2.5. Let $\left\{f_{1 / k}\right\}_{k \geq 1}$ be the approximating sequence of Lemma 2.4 generated by $f \in \mathcal{F}_{r}$. Since $f \leq 0$ in $B_{c}(0, r)$ and the smoothing kernel $\kappa$ is a nonnegative function, $f_{1 / k} \leq 0$ in $B_{c}(0, r-1 / k)$. Moreover, $\left\{f_{1 / k}(z)\right\}_{k \geq 1}$ converges monotonically to $f(z)$ at any $z \in B_{c}(0, r)$. Then for $k$ sufficiently large, say $k \geq k_{0}$,

$$
\sup _{B_{c}(0,1)} f_{1 / k} \geq \sup _{B_{c}(0,1)} f \geq-1 \text {. }
$$

Thus $f_{1 / k} \in C^{\infty} \cap \mathcal{F}_{r-1 / k}, k \geq k_{0}$. We now set $r_{k}:=r-1 / k$ and consider the sequence

$$
\left\{\sup _{B_{c}\left(x, \frac{r_{k}-|x|}{r_{k}}\right)} f_{1 / k}\right\}_{k \geq k_{0}} .
$$

From Lemma 2.6 it follows that

$$
\sup _{B_{c}\left(x, \frac{r_{k}-|x|}{r_{k}}\right)} f_{1 / k}>2 C(r)
$$

for $k \geq k_{1}\left(\geq k_{0}\right)$. Since $f_{1 / k} \in C^{\infty} \cap \mathcal{P} \mathcal{S H}\left(B_{c}\left(0, r_{k}\right)\right)$, there is a point $z \in$ $\partial B_{c}\left(x, \frac{r_{k}-|x|}{r_{k}}\right)$ where the supremum is attained. Further, there is an open neighborhood $U$ of $z$ where $f_{1 / k}$ is greater than $2 C(r)$. Thus there exists a finite family $\mathcal{G}$ of rotations (unitary transformations) of $\mathbb{C}^{n}$ centered at $x$ such that $\{g(U)\}_{g \in \mathcal{G}}$ forms a covering of an open neighborhood $W$ of $\partial B_{c}\left(x, \frac{r_{k}-|x|}{r_{k}}\right)$. The plurisubharmonic function

$$
g_{k}(x):=\max _{g \in \mathcal{G}} f_{1 / k}(g x) \quad\left(k \geq k_{1}\right)
$$

satisfies

$$
\max _{B_{c}(x, s)} g_{k}=\max _{B_{c}(x, s)} f_{1 / k}
$$

for any $s \in\left(0, r_{k}-|x|\right)$ and

$$
g_{k}(w)>2 C(r)
$$

for any $w \in \partial B_{c}\left(x, \frac{r_{k}-|x|}{r_{k}}\right)$. 
Now set

$$
c_{k}:=\frac{2 C(r)}{\log \frac{r+1}{2 r_{k}}} .
$$

Since $r>1$, this constant is greater than 0 for $k$ sufficiently large, and we can assume that it holds for $k \geq k_{1}$. We now define for $k \geq k_{1}$ the function

$$
h_{k}(z):=\left\{\begin{array}{cc}
g_{k}(z) & \left(z \in B_{c}\left(x, \frac{r_{k}-|x|}{r_{k}}\right)\right) \\
\max \left\{c_{k} \log \frac{(r+1)|z-x|}{2\left(r_{k}-|x|\right)}, g_{k}(z)\right\} & \left(z \in B_{c}\left(x, r_{k}-|x|\right) \backslash B_{c}\left(x, \frac{r_{k}-|x|}{r_{k}}\right)\right) \\
c_{k} \log \frac{(r+1)|z-x|}{2\left(r_{k}-|x|\right)} & \left(z \notin B_{c}\left(x, r_{k}-|x|\right)\right) .
\end{array}\right.
$$

Since the logarithmic term is less than $g_{k}$ on $\partial B_{c}\left(x, \frac{r_{k}-|x|}{r_{k}}\right)$, by (ii) above, and more than 0 on $\partial B_{c}\left(x, r_{k}-|x|\right)$ since $r>1$, the function $h_{k}$ is plurisubharmonic on $\mathbb{C}^{n}$. Furthermore, the function $h_{k}^{\prime}=\frac{1}{c_{k}} h_{k}$ clearly belongs to $\mathcal{L}\left(\mathbb{C}^{n}\right)$. Compare now $h_{k}^{\prime}$ with the global $\mathcal{L}$-extremal function $E_{B_{c}(x, t)}$ defined by

$$
E_{B_{c}(x, t)}(z):=\sup \left\{u(z): u \in \mathcal{L}\left(\mathbb{C}^{n}\right), u \leq 0 \text { on } B_{c}(x, t)\right\}
$$

From this definition it follows that

$$
h_{k}^{\prime}-\sup _{B_{c}(x, t)} h_{k}^{\prime} \leq E_{B_{c}(x, t)} .
$$

Now we make use of the important representation of $\mathcal{L}$-extremal functions (see, e.g., [K, Th. 5.1.7]),

$$
E_{B_{c}(x, t)}(z):=\sup \left\{\frac{\log |P(z)|}{\operatorname{deg} P} ; \quad \max _{B_{c}(x, t)}|P| \leq 1\right\},
$$

where the supremum is taken over all holomorphic polynomials on $\mathbb{C}^{n}$. This representation and the classical Bernstein inequality on the growth of univariate complex polynomials lead to the estimate

$$
\sup _{B_{c}(x, s t)} E_{B_{c}(x, t)} \leq \log s \quad(1 \leq s) .
$$

From (2.25) it follows that

$$
\sup _{B_{c}(x, s t)} h_{k}-\sup _{B_{c}(x, t)} h_{k} \leq c_{k} \log s .
$$

From property (i) of $g_{k}$ and the definition of $h_{k}$, the doubling inequality (2.15) is obtained for $f_{1 / k}$ with the constant $c_{k}, k \geq k_{1}$. Applying Lemma 2.4 we obtain then the doubling inequality for $f \in \mathcal{F}_{r}$ with $c=\frac{2 C(r)}{\log \frac{r+1}{2 r}}$. 
3. Proof of the Yu. Brudnyĭ-Ganzburg type inequality. We have to prove that if $f \in \mathcal{F}_{r}$ and $\omega$ is a measurable subset of $B(x, t)\left(\subset B_{c}(x, a t) \subset B_{c}(0,1)\right)$ of positive measure then

$$
\sup _{B(x, t)} f \leq c \log \frac{d|B(x, t)|}{|\omega|}+\sup _{\omega} f
$$

It is clear that we may assume that $\omega$ is compact. In fact, otherwise $\omega=$ $\omega_{0} \cup\left(\bigcup_{j=1}^{\infty} \omega_{j}\right)$, where $\left|\omega_{0}\right|=0$ and $\left\{\omega_{j}\right\}$ is an increasing sequence of compact sets. If (2.27) holds for every $\omega_{j}$, then we obtain the result for $\omega$ by letting $j \rightarrow \infty$ since $c=c(a, r)$ and $d=d(n)$ do not depend on $\omega$.

Taking into account Proposition 2.5 and the approximation of Lemma 2.4, it suffices to prove the following equivalent statement. Let $B_{c}(0,1) \subset B_{c}(0, a)$ and $\mathcal{R}_{a}$ be the family of continuous plurisubharmonic functions $f: B_{c}(0, a) \longrightarrow$ $\mathbb{R}$ satisfying

$$
\begin{aligned}
& \sup _{B_{c}(0, a)} f=0 \\
& \sup _{B_{c}(0,1)} f \geq-c \log a,
\end{aligned}
$$

with the constant $c$ from Proposition 2.5. Then for every measurable subset $\omega \subset B(0,1)$ of positive measure and every $f \in \mathcal{R}_{a}$,

$$
\sup _{B(0,1)} f \leq c^{\prime} \log \frac{d|B(0,1)|}{|\omega|}+\sup _{\omega} f
$$

Here $d=d(n)$ and $c^{\prime}=c^{\prime}(a, c)$. In fact, by a translation and a dilation with coefficient $\frac{1}{t}$ we can transform the balls $B_{c}(x, t)$ and $B_{c}(x, a t)$ into the balls $B_{c}(0,1)$ and $B_{c}(0, a)$, respectively. Then the first term on the right in $(2.27)$ does not change. Moreover, the inequality of Proposition 2.5 states that the pullback of a function $f \in \mathcal{F}_{r}$ determined by this transformation will satisfy to conditions (i) and (ii). Finally, according to Lemma 2.4 we can assume that $f$ is continuous on $B_{c}(0,1)$.

It remains to prove $(2.28)$. We begin with

Lemma 2.7. There is a constant $C=C(c, a)>0$ such that

$$
\max _{B(0,1)} f \geq-C
$$

for every $f \in \mathcal{R}_{a}$. 
Proof. We can repeat the arguments of Lemma 2.6 related to the use of the relative extremal function $(2.20)$. In this case the ball $B(0,1)$ is nonpluripolar. So using the inequality from (ii) we obtain (2.29) with, e.g., $C=\frac{c \log a}{|M(a)|}$, where

$$
M(a):=\sup _{\partial B_{c}(0,(1+a) / 2)} u_{B(0,1), B_{c}(0, a)} .
$$

Now let $f \in \mathcal{R}_{a}$ and $x_{f} \in B(0,1)$ be such that

$$
M_{f}:=f\left(x_{f}\right)=\max _{B(0,1)} f
$$

By Lemma 3 of $[\mathrm{BG}]$ there is a ray $l_{f}$ with origin at $x_{f}$ such that

$$
\frac{\operatorname{mes}_{1}\left(B(0,1) \bigcap l_{f}\right)}{\operatorname{mes}_{1}\left(\omega \cap l_{f}\right)} \leq \frac{n|B(0,1)|}{|\omega|} .
$$

Let $l_{f}^{\prime}$ be the one-dimensional affine complex line containing $l_{f}$ and let $z_{f}$ be a point of $B_{c}(0,1) \cap l_{f}^{\prime}$ such that

$$
t_{f}:=\operatorname{dist}\left(0, l_{f}^{\prime}\right)=\left|z_{f}\right|
$$

Consider the disks

$$
\widetilde{D}_{f}:=\frac{1}{r_{f}}\left(D_{f}-z_{f}\right) \text { and } \quad \widetilde{D}_{f}^{\prime}:=\frac{1}{r_{f}}\left(D_{f}^{\prime}-z_{f}\right)
$$

where we set

$$
D_{f}:=l_{f}^{\prime} \cap B_{c}(0, a), \quad D_{f}^{\prime}:=l_{f}^{\prime} \cap B_{c}(0,(a+1) / 2) .
$$

The latter sets are disks of radii

$$
r_{f}:=\sqrt{a^{2}-t_{f}^{2}}, \quad r_{f}^{\prime}:=\sqrt{\left(\frac{a+1}{2}\right)^{2}-t_{f}^{2}},
$$

respectively centered at $z_{f}$. Note also that $x_{f} \in D_{f}^{\prime}$. Without loss of generality we can identify $\widetilde{D}_{f}^{\prime} \subset \widetilde{D}_{f}$ with the pair $\mathbb{D}_{s_{f}} \subset \mathbb{D}_{1}$, where $\mathbb{D}_{r}:=\{z \in \mathbb{C} ;|z| \leq r\}$ and $s_{f}:=r_{f}^{\prime} / r_{f}$. The pullback of the restriction $\left.f\right|_{D_{f}}$ to $\mathbb{D}_{1}$ is denoted by $f^{\prime}$.

LEMMA 2.8. There exists a number $r=r(a)<1$ such that

$$
\mathbb{D}_{s_{f}} \subset \mathbb{D}_{r} \subset \mathbb{D}_{1}
$$

for any $f \in \mathcal{R}_{a}$.

Proof. It follows from the inequality

$$
\frac{r_{f}^{\prime}}{r_{f}}=\sqrt{\frac{(a+1)^{2}-4 t_{f}^{2}}{4\left(a^{2}-t_{f}^{2}\right)}} \leq \frac{a+1}{2 a}<1
$$

that one can choose $r(a)=\frac{a+1}{2 a}$. 
In what follows it is worth noting that

$$
\max _{\mathbb{D}_{r}} f^{\prime} \geq \max _{\mathbb{D}_{s_{f}}} f^{\prime} \geq M_{f} \geq-C
$$

by $(2.30)$ and $(2.29)$. We apply now Proposition 2.2 to the function $\frac{f^{\prime}}{C}$ which clearly satisfies condition (2.2), i.e., belongs to $\mathcal{A}_{r}$. Returning to the function $f$ we can formulate the result of this proposition as follows.

Statement. There exists a subharmonic function $h_{f}$ defined on $l_{f}^{\prime}$ and a constant $c_{f}>0$ such that $h_{f} / c_{f}$ is of minimal growth on $l_{f}^{\prime}$ and $f=h_{f}$ on $D_{f}^{\prime}$. Moreover,

$$
c^{\prime}=c^{\prime}(c, a):=\sup _{f \in \mathcal{R}_{a}} c_{f}<\infty .
$$

Set $m_{f}(\omega):=\max _{\omega \cap l_{f}} f$ and consider the function $\frac{h_{f}-m_{f}(\omega)}{c_{f}}$. This function is clearly less than or equal to the $\mathcal{L}$-extremal function $E_{\omega \cap l_{f}}$ (see $(2.24)$ for the definition, replacing $\mathcal{L}\left(\mathbb{C}^{n}\right)$ by $\mathcal{L}\left(l_{f}\right)$ and $B_{c}(x, t)$ by $\left.\omega \cap l_{f}\right)$. Using the polynomial representation $(2.26)$ of $\mathcal{L}$-extremal functions and the one-dimensional Yu. Brudnyǐ-Ganzburg inequality (see the remark after (1.4) and (2.31)), we obtain

$$
\max _{B(0,1) \cap l_{f}} E_{\omega \cap l_{f}} \leq \log \frac{4 \operatorname{mes}_{1}\left(B(0,1) \cap l_{f}\right)}{\operatorname{mes}_{1}\left(\omega \cap l_{f}\right)} \leq \log \frac{4 n|B(0,1)|}{|\omega|} .
$$

From the selection of $l_{f}$ and the statement above, we obtain

$$
\max _{B(0,1)} f-\max _{\omega} f \leq \max _{B(0,1) \cap l_{f}} h_{f}-m_{f}(\omega) \leq c_{f} \max _{B(0,1) \cap l_{f}} E_{\omega \cap l_{f}} \leq c^{\prime} \log \frac{4 n|B(0,1)|}{|\omega|} .
$$

This proves the desired inequality (2.28) for $f \in \mathcal{R}_{a}$ and, hence, Theorem 1.2.

\section{Proof of consequences}

1. Proof of Theorem 1.3. Let $V \subset \mathbb{R}^{n}$ be a real algebraic variety of pure dimension $m, 1 \leq m \leq n-1$. Let $x \in V$ be a regular point of $V$. We have to prove that there is an open neighbourhood $N \subset V$ of $x$ depending on $V$ such that

$$
\sup _{B}|p| \leq\left(\frac{d \lambda_{V}(B)}{\lambda_{V}(\omega)}\right)^{\alpha k \operatorname{deg}(V)} \sup _{\omega}|p|
$$

for every ball $B \subset N$, measurable subset $\omega \subset B$ and real polynomial $p$ of degree $k$. Here $\operatorname{deg}(V)$ is the degree of the manifold $V, d=d(m)$ depends only on $\operatorname{dim} V$ and $\alpha$ is an absolute constant. 
For the proof we need an estimate for a $p$-valent function regular in $\mathbb{D}_{R}$ $:=\{z \in \mathbb{C} ;|z|<R\}$. This estimate is due to Roytwarf and Yomdin (see [RY, Th. 2.1.3 and Cor. 2.3.1]). We give, for the sake of completeness, a relatively simple proof of the result.

Lemma 3.1. Suppose that $f: \mathbb{D}_{R} \longrightarrow \mathbb{C}$ is regular and assumes no value more than $p$ times. Then for any $R^{\prime}<R$ and any $\alpha \in(0,1)$ the inequality

$$
\max _{\mathbb{D}_{R^{\prime}}}|f| \leq C^{p} \max _{\mathbb{D}_{\alpha R^{\prime}}}|f|
$$

holds with $C=C(\alpha, \beta)$, where $\beta:=\frac{R^{\prime}}{R}$.

Proof. We set

$$
M:=\max _{\mathbb{D}_{R^{\prime}}}|f| \quad \text { and } \quad M_{\alpha}:=\max _{\mathbb{D}_{\alpha R^{\prime}}}|f| .
$$

If $f(z)=\sum_{k=0}^{\infty} a_{k} z^{k}$ then

$$
M \leq \sum_{k=0}^{p}\left|a_{k}\right|\left(R^{\prime}\right)^{k}+\sum_{k=p+1}^{\infty}\left|a_{k}\right|\left(R^{\prime}\right)^{k}=: \sum_{1}+\sum_{2} .
$$

From the Cauchy inequality for $\mathbb{D}_{\alpha R^{\prime}}$ we get

$$
\sum_{1} \leq M_{\alpha} \sum_{k=0}^{p}\left(\frac{R^{\prime}}{\alpha R^{\prime}}\right)^{k} \leq \frac{\alpha^{-p}}{1-\alpha} M_{\alpha}
$$

To estimate the second sum we apply the coefficient inequality for $p$-valent functions (see $[\mathrm{H}]$ ). According to it,

$$
\left|a_{j}\right| R^{j} \leq\left(\frac{A}{p}\right)^{2 p} j^{2 p} \max _{1 \leq j \leq p}\left|a_{j}\right| R^{j}
$$

for every $j>p$, where $A$ is an absolute constant.

By the Cauchy inequality the maximum on the right of (3.4) can be estimated by

$$
M_{\alpha} \max _{1 \leq j \leq p} \frac{R^{j}}{\left(\alpha R^{\prime}\right)^{j}} \leq M_{\alpha}(\alpha \beta)^{-p} .
$$

Putting this and (3.4) together we obtain

$$
\sum_{2} \leq\left(\frac{A}{p}\right)^{2 p}(\alpha \beta)^{-p} M_{\alpha} \sum_{j=p+1}^{\infty} j^{2 p}\left(\frac{R^{\prime}}{R}\right)^{j} \leq\left(\frac{A}{p}\right)^{2 p}(\alpha \beta)^{-p} \phi_{2 p}(\beta) M_{\alpha},
$$

where

$$
\phi_{l}(\beta)=\sum_{k=1}^{\infty} k^{l} \beta^{k}
$$


We will prove later that

$$
\phi_{2 p}(\beta)<\frac{(4 p)^{2 p}}{(1-\beta)^{2 p+1}}
$$

which together with the previous inequality leads to the estimate

$$
\sum_{2} \leq(4 A)^{2 p}(\alpha \beta)^{-p}(1-\beta)^{-2 p-1} M_{\alpha}
$$

From this and (3.3), the required inequality (3.2) follows with

$$
C(\alpha, \beta)=2 \max \left\{\frac{1}{\alpha(1-\alpha)}, \frac{(4 A)^{2}}{\alpha \beta(1-\beta)^{3}}\right\} .
$$

It remains to prove (3.5). To this end, notice that

$$
\phi_{l}(\beta)=\left(\beta \frac{d}{d \beta}\right)^{l}\left(\frac{1}{1-\beta}\right) .
$$

Then by induction on $l$ we have

$$
\phi_{l}(\beta)=\frac{p_{l}(\beta)}{(1-\beta)^{l+1}},
$$

where $p_{l}$ is a polynomial of degree $l$. Moreover, we have the identity

$$
p_{l+1}(\beta)=\beta(1-\beta) p_{l}^{\prime}(\beta)+(l+1) p_{l}(\beta) .
$$

Let $\mu(l):=\max _{0 \leq \beta \leq 1}\left|p_{l}(\beta)\right|$. Then from the previous identity and the Bernstein polynomial inequality we get

$\mu(l+1) \leq \max _{0 \leq \beta \leq 1}\left|\beta(1-\beta) p_{l}^{\prime}(\beta)\right|+(l+1) \mu(l) \leq l \mu(l)+(l+1) \mu(l)=(2 l+1) \mu(l)$.

This recurrence inequality implies that

$$
\mu(2 p) \leq \mu(0) \prod_{l=0}^{2 p-1}(2 l+1)=\prod_{l=0}^{2 p-1}(2 l+1)<(4 p)^{2 p},
$$

which combined with (3.6) gives the required estimate (3.5).

We now proceed to the proof of Theorem 1.3. Let $V_{c}$ denote the complexification of $V$, i.e., the minimal complex algebraic subvariety of $\mathbb{C}^{n}$ such that $V$ is a connected component of $V_{c} \cap \mathbb{R}^{n}$. Then the regularity of $x$ in $V$ implies that it is a regular point of $V_{c}$. We will assume without loss of generality that $x$ coincides with the origin $0 \in \mathbb{R}^{n}$. Then there exist open neighborhoods $U_{x} \subset U_{x}^{\prime} \subset V_{c}$ of the point $x$ and a linear holomorphic projection of $\mathbb{C}^{n}$ onto $\mathbb{C}^{m}$ whose restriction $\phi: V_{c} \longrightarrow \mathbb{C}^{m} \subset \mathbb{C}^{n}$ is biholomorphic in a neighborhood of $U_{x}^{\prime}$ such that

(i) $\phi(x)=0, \quad \phi\left(U_{x}\right)=B_{c}(0, r), \quad$ and $\quad \phi\left(U_{x}^{\prime}\right)=B_{c}(0,2 r)$ for some $r>0$;

(ii) $\left.\phi\right|_{V}: V \longrightarrow \mathbb{R}^{m}$ 
(see, e.g., [Br, Prop. 2.8]). According to (ii), $\phi$ has a smooth inverse defined on $B(0, r)$ and $N^{\prime}:=\phi^{-1}(B(0, r))$ is an open neighborhood of $x$ in $V$.

Consider now a real polynomial $p \in \mathcal{P}_{k, n}$ and its extension $p_{c}$ to $\mathbb{C}^{n}$ as a holomorphic polynomial of degree $k$. Let $z \in B_{c}(0, r)$ be a maximum point of $\left|p_{c} \circ \phi^{-1}\right|$ in $B_{c}(0, r)$. Let $L$ be a complex line passing through $z$ and the origin. The restriction of $p_{c} \circ \phi^{-1}$ to $L$ is an algebraic function of one variable, whose local valency can be estimated by the multidimensional Bezout theorem. By this theorem the function $p_{c} \circ \phi^{-1}$ assumes no value more than $p:=k \operatorname{deg}\left(V_{c}\right)$ times in $B_{c}(0,2 r) \cap L$. Applying Lemma 3.1 to this function we obtain

$$
M_{r}:=\max _{B_{c}(0,3 r / 2)}\left|p_{c} \circ \phi^{-1}\right| \leq C^{k \operatorname{deg}(V)} \max _{B_{c}(0, r)}\left|p_{c} \circ \phi^{-1}\right|,
$$

where $C$ is an absolute constant.

Consider now the function $f: B_{c}(0,3 / 2) \longrightarrow \mathbb{R}$ defined by

$$
f(z):=\frac{1}{k \log c \operatorname{deg}(V)}\left(\log \left|\left(p_{c} \circ \phi^{-1}\right)(r z)\right|-\log M_{r}\right) .
$$

Then $\sup _{B_{c}(0,3 / 2)} f=0$ and, by (3.7), $\sup _{B_{c}(0,1)} f \geq-1$. So $f \in \mathcal{F}_{3 / 2}$ and the conditions of Theorem 1.2 are fulfilled. Applying Theorem 1.2 to $f$ we get

$$
\sup _{\phi(B)}\left|p \circ \phi^{-1}\right| \leq\left(\frac{d(m)|\phi(B)|}{|\phi(\omega)|}\right)^{\alpha k \operatorname{deg}(V)} \sup _{\phi(\omega)}\left|p \circ \phi^{-1}\right|
$$

for every ball $\phi(B) \subset B(0, r)$ and measurable subset $\omega \subset B$. Here $\alpha:=c \log C$, where $c$ and $d(m)$ are the constants in the inequality of Theorem 1.2 and $C$ is that of (3.7). To finish the proof we note that the metric in $U_{x} \cap V$ induced from $\mathbb{R}^{n}$ is equivalent to the metric lifted from $B_{c}(0,3 r / 2)$ by means of $\phi$. Therefore we can find a smaller neighborhood $N \subset N^{\prime}$ of the point $x$ (depending on $\phi$ ) in which these two metrics are Lipschitz equivalent with the coefficient 2. Hence (3.8) will be valid for a metric ball $B \subset N$ and a measurable subset $\omega \subset B$, replacing $\phi(B)$ and $\phi(\omega)$, respectively, with $C(m)>d(m)$ replacing $d(m)$, and with the measure induced from $\mathbb{R}^{n}$. The proof is complete.

2. Proof of Theorem 1.4. According to the Bernstein doubling theorem of [FN3] and the Hadamard three circle theorem we have under the assumptions of Theorem 1.4 the following inequality.

For any compact $K \subset U$ and $F \in V_{\lambda}, \lambda \in K$,

$$
\sup _{B_{c}(0, r)}|F| \leq C_{1} \sup _{B_{c}(0,1)}|F|,
$$

where $r=\frac{2+r_{0}}{2}$ and $C_{1}=C_{1}\left(K, r_{0}\right)$. Then the function

$$
F^{\prime}:=\frac{1}{\log C_{1}}\left(\log |F|-\sup _{B_{c}(0, r)} \log |F|\right)
$$


belongs to $\mathcal{F}_{r}$. It remains to apply the inequality of Theorem 1.2 to obtain the required inequality

$$
\sup _{B(x, \rho)}|F| \leq\left(\frac{d(n)|B(x, \rho)|}{|\omega|}\right)^{\gamma} \sup _{\omega}|F| \quad(\rho \leq 1)
$$

with $\gamma:=c \log C_{1}$, where $c$ and $d(n)$ are the constants in Theorem 1.2.

3. Proof of Theorems 1.5 and 1.7. Let $(Y, \mu)$ be a compact set with a positive Borel measure $\mu$. Assume that $f: Y \longrightarrow \mathbb{C}$ is a not-identically-0 continuous function satisfying

$$
\|f\|_{Y}:=\max _{Y}|f| \leq\left(\frac{C \mu(Y)}{\mu(\omega)}\right)^{\alpha} \sup _{\omega}|f|
$$

for every measurable set $\omega \subset Y$. Here $\alpha$ and $C$ are constants. Then $f$ satisfies

$$
\frac{1}{\mu(Y)} \int_{Y}\left|\log \frac{|f|}{\|f\|_{Y}}\right| d \mu \leq \alpha C^{\prime}
$$

with $C^{\prime}=C^{\prime}(C)$. The proof of (3.11) repeats word-for-word the arguments used in Theorem 5.1 of $[\mathrm{Br}]$.

We now prove Theorem 1.5. According to Theorem 1.3, for every $x$ in the $m$-dimensional compact algebraic manifold $V \subset \mathbb{R}^{n}$ there exists a ball $B_{r(x)}(x)$ such that

$$
\sup _{B}|p| \leq\left(\frac{d(m) \lambda_{V}(B)}{\lambda_{V}(\omega)}\right)^{\alpha k \operatorname{deg}(V)} \sup _{\omega}|p|
$$

for every ball $B \subset B_{r(x)}(x)$ and polynomial $p \in \mathcal{P}_{k, n}$ with $\left.p\right|_{V} \not \equiv 0$. Recall that $\lambda_{V}$ denotes the measure on $V$ induced by the Lebesgue measure of $\mathbb{R}^{n}$ and $B_{\rho}(y)$ is the metric ball of radius $\rho$ centered at $y$.

Let $\left\{B^{l}:=B_{r\left(x_{l}\right)}\left(x_{l}\right)\right\}_{l=1}^{s}$ be a finite subcovering of the covering $\left\{B_{r(x)}(x)\right\}_{x \in V}$, and let $\mu$ be its multiplicity. According to the Lebesgue theorem there exists a constant $r_{0}>0$ such that every ball $B \subset V$ of radius less than $r_{0}$ is contained in one of the $B^{l}$. So inequality (3.12) holds for such a ball with the exponent $\alpha k \operatorname{deg}(V)$. Applying inequality (3.11) to $|p|$ and $Y:=\bar{B}$ we get from (3.12)

$$
\frac{1}{\lambda_{V}(B)} \int_{B}\left|\log \frac{|p|}{\|p\|_{B}}\right| d \lambda_{V} \leq C k \operatorname{deg}(V)
$$

with $C=C(m, \alpha)$.

Let us now prove that (3.13) holds also for balls of radii greater than $r_{0}$. First, we note that for any ball $B$ of radius greater than or equal to $r_{0}$ there is a constant $m=m\left(V, r_{0}\right)$ such that

$$
\lambda_{V}(B) \geq m
$$


Furthermore, from this it follows that

$$
\begin{aligned}
& \frac{1}{k \lambda_{V}(B)} \int_{B}\left|\log \frac{|p|}{\|p\|_{B}}\right| d \lambda_{V} \\
& \quad \leq \frac{1}{k m} \sum_{l=1}^{s}\left(\int_{B^{l}}\left|\log \frac{|p|}{\|p\|_{B^{l}}}\right| d \lambda_{V}+\log \frac{\|p\|_{V}}{\|p\|_{B^{l}}} \lambda_{V}\left(B^{l}\right)\right) .
\end{aligned}
$$

Applying now the Bernstein-Walsh inequality (see, e.g., $[\mathrm{K}]$ ), we estimate the second term on the right-hand side of the inequality above as follows:

$$
\frac{1}{k} \log \frac{\|p\|_{V}}{\|p\|_{B^{l}}} \leq \sup _{V} E_{B^{l}}=: C_{l},
$$

where $E_{B^{l}}$ is the global $\mathcal{L}$-extremal function of $B^{l}$ (see (2.26)). Since $B^{l} \subset V_{c}$ is not pluripolar, Theorem 2.2 of [S] shows that $C_{l}<\infty$. Putting together the two previous inequalities and (3.13) for $B^{l}$ we obtain

$$
\frac{1}{k \lambda_{V}(B)} \int_{B}\left|\log \frac{|p|}{\|p\|_{B}}\right| d \lambda_{V} \leq \frac{\mu \operatorname{vol}(V)}{m}\left(C \operatorname{deg}(V)+\max _{1 \leq l \leq s} C_{l}\right)=: C^{\prime} .
$$

From this inequality and (3.13) it follows that the BMO-norm of $\log |p|$ is bounded above by $C(V) k$, where $C(V):=2 \max \left(C^{\prime}, C \operatorname{deg}(V)\right)=2 C^{\prime}$. The proof of Theorem 1.5 is complete.

The proof of Theorem 1.7 is along the same lines as the previous one, so we will only give a sketch of it. Let $V$ be a compact real analytic manifold of real dimension $n$. Let $\left\{F_{s, \lambda}\right\}_{s=1}^{N}$ be a family of real analytic functions on $V$ depending real-analytically on $\lambda$ in an open subset $U \subset \mathbb{R}^{m}$, and let $V_{\lambda}:=$ $\operatorname{span}\left\{F_{s, \lambda}\right\}$. By the principle of analytic extension, for each $x \in V$ and $\lambda_{0} \in K$ there exist an open neighborhood $U_{x} \times W_{\lambda_{0}, x}$ of the point $\left(x, \lambda_{0}\right) \in V \times U$ and an analytic embedding $\phi_{x, \lambda_{0}}: U_{x} \times W_{\lambda_{0}, x} \longrightarrow B_{c}^{n}(0,1) \times B_{c}^{m}(0,1) \subset \mathbb{C}^{n} \times \mathbb{C}^{m}$ such that

$$
\phi_{x, \lambda_{0}}\left(U_{x} \times W_{\lambda_{0}, x}\right)=\left(B_{c}^{n}(0,1) \cap \mathbb{R}^{n}\right) \times\left(B_{c}^{m}(0,1) \cap \mathbb{R}^{m}\right) .
$$

Moreover, each $\left(\phi^{-1}\right)^{*}\left(F_{s,}\right), 1 \leq s \leq N$, admits a unique holomorphic extension to $B_{c}^{n}(0,1) \times B_{c}^{m}(0,1)$. Diminishing, if necessary, the neighborhood $U_{x} \times W_{\lambda_{0}, x}$ we can apply Theorem 1.4 to functions from the linear span of the extended family $\left\{F_{s,}^{c}\right\}$. Then returning to the coordinates on $V$ we determine that the inequality

$$
\sup _{B}|F| \leq\left(\frac{C(n) \lambda_{V}(B)}{\lambda_{V}(\omega)}\right)^{c_{x}} \sup _{\omega}|F|
$$

holds for every $F \in V_{\lambda}$ with $\lambda \in W_{\lambda_{0}, x}$ and for every measurable subset $\omega$ of a metric ball $B \subset U_{x}$. 
For a fixed $\lambda_{0} \in K$ consider the open covering $\left\{U_{x}\right\}_{x \in V}$ of $V$, and select a finite subcovering $\left\{U_{s\left(\lambda_{0}\right)}:=U_{x_{s}}\right\}_{s=1}^{s\left(\lambda_{0}\right)}$. We set now

$$
\widetilde{W}_{\lambda_{0}}:=\bigcap_{s=1}^{s\left(\lambda_{0}\right)} W_{\lambda_{0}, x_{s}} .
$$

As in the proof of Theorem 1.5, we derive from (3.14) the inequality

$$
\frac{1}{\lambda_{V}(B)} \int_{B}\left|\log \frac{|F|}{\|F\|_{B}}\right| d \lambda_{V} \leq C\left(V, \lambda_{0}\right)
$$

for small metric balls $B$, where $F \in V_{\lambda}$ and $\lambda \in \widetilde{W}_{\lambda_{0}}$. To estimate this quantity for large balls we need only use the Bernstein doubling inequality from [FN3] instead of the Bernstein-Walsh inequality which has been used in the proof of Theorem 1.5. In this way we obtain the required estimate of the BMO-norm $|\log | F \|_{*}$ for $F \in V_{\lambda}, \lambda \in \widetilde{W}_{\lambda_{0}}$. Finally, taking a finite subcovering $\left\{\widetilde{W}_{\lambda_{i}}\right\}_{i=1}^{s}$ of the covering $\left\{\widetilde{W}_{\lambda_{0}}\right\}_{\lambda_{0} \in K}$ and using the finite number of the estimates corresponding to $\widetilde{W}_{\lambda_{i}}, 1 \leq i \leq s$, we get the global estimate of $|\log | F \mid \|_{*}$ for any $F \in V_{\lambda}$ with an arbitrary $\lambda \in K$. The proof is complete.

\section{Concluding remarks}

1. One can generalize the local inequality for polynomials of Theorem 1.3 as follows.

TheOREM 4.1. For every regular point $x \in V$ there exists an open neighborhood $N=N(V)$ of $x$ such that for every ball $B \subset N$, measurable subset $\omega \subset B$, and polynomial $p \in \mathcal{P}_{k, n}(\mathbb{R})$ we have

$$
\sup _{B}|p| \leq(\alpha k \operatorname{deg}(V)+1)\left(\frac{d(m) \lambda_{V}(B)}{\lambda_{V}(\omega)}\right)^{\alpha k \operatorname{deg}(V)}\left(\frac{1}{\lambda_{V}(\omega)} \int_{\omega}|p| d \lambda_{V}\right) .
$$

Here $N, \alpha$, and $d(m)$ are as in Theorem 1.3.

To prove the result it suffices to use an inequality for the distribution function of $\left.p\right|_{V}$ similar to the inequality of Theorem 1.2 (see [BG] for details).

2. It was discovered in $[\mathrm{BM}]$ and [FN2], independently and in different ways that local Bernstein-type inequalities can be obtained from the Hadamard three circles theorem. Based on this idea, it was proved in [FN2] that Markov's inequality for algebraic functions with the constant depending on $(\operatorname{deg} p)^{2}$ linearly can be derived from the classical one-dimensional doubling Bernstein inequality. We remark that by applying a suitable version of Hadamard's theorem for the case of three polydisks one can deduce the required Markov inequality from the doubling inequality of Theorem 1.3 in a straightforward manner. 
In the general case we can state that under the assumptions and notation of Theorem 1.3

$$
\sup _{B}|\nabla p| \leq \frac{1}{r}\left(\frac{d(m) \lambda_{V}(B)}{\lambda_{V}(\omega)}\right)^{k \gamma}\left(\frac{1}{\lambda_{V}(\omega)} \int_{\omega}|p| d \lambda_{V}\right)
$$

for a $p \in \mathcal{P}_{k, n}(\mathbb{R})$. Here $r$ is radius of $B$ and $\gamma=\gamma(N)$.

3. The BMO-properties of Theorems 1.5 and 1.7 produce a large class of examples of functions from BMO. Since a quasi-conformal change of coordinates preserves the BMO-class one can obtain additional examples.

The arguments based on the inequality of Theorem 1.2 allow us also to prove the following result:

For any real analytic functions $f_{1}, \ldots, f_{k}$ defined on a compact real analytic manifold $V$ the function $\max _{i} \log \left|f_{i}\right|$ belongs to $\operatorname{BMO}(V)$.

Using, in addition, Proposition 2.2 we can state

For any subharmonic function $f \not \equiv-\infty$ defined in an open neighborhood of the unit circle $S^{1}$ the restriction $\left.f\right|_{S^{1}}$ belongs to $\operatorname{BMO}\left(S^{1}\right)$.

4. Inequality (1.2) can be used also in the problem, first posed by B. Panejah [P] for $L_{2}\left(\mathbb{R}^{n}\right)$, on the equivalence of $L_{p}$-norms of entire functions of exponential type over $\mathbb{R}^{n}$ and a relatively dense subset.

Definition 4.2. A measurable subset $E \subset \mathbb{R}^{n}$ is said to be relatively dense if there is a constant $L>0$ such that

$$
\delta:=\inf _{x \in \mathbb{R}^{n}} \operatorname{mes}(B(x, L) \cap E)>0 .
$$

THEOREM 4.3. Let $E \subset \mathbb{R}^{n}$ be relatively dense (with constants $L$ and $\delta$ ) and $u: \mathbb{C}^{n} \longrightarrow \mathbb{R} \cup\{-\infty\}$ be a plurisubharmonic function satisfying

$$
u(z) \leq \sigma(1+|y|)+N|x|+c
$$

for some $\sigma, N \geq 0$ and $c \in \mathbb{R}$. Then

$$
\sup _{x \in \mathbb{R}^{n}} u(x) \leq C^{\prime} \sigma L \log \frac{c(n) L^{n}}{\delta}+\sup _{x \in E} u(x) .
$$

Here $C^{\prime}$ is an absolute constant, $z=x+i y$ and $|\cdot|$ denotes the Euclidean norm.

Comparing this result with similar inequalities for $p$-subharmonic functions due to B. Ya. Levin and V. N. Logvinenko [LL], we note that for $n=1$ (where both inequalities are the same) our approach leads to the improved constant $C \sigma L \log (4 L / \delta)$ instead of $C \sigma L^{2} / \delta$ in [LL]. We have to stress, nevertheless, that our proof uses an important component of the proof in [LL] due to B. Ya. Levin. 
In a forthcoming paper we present the proof of Theorem 4.3 and its generalization for regular coverings over compact algebraic manifolds (e.g. $\mathbb{R}^{n}$ is the covering over an $n$-torus).

The author thanks Professors Yu. Brudnyǐ and N. Levenberg for inspiring discussions.

University of Toronto, Toronto, Canada

E-mail address: brudnyi@math.toronto.edu

\section{REFERENCES}

[AT] H. Alexander and B. A. Taylor, Comparison of two capacities in $\mathbb{C}^{N}$, Math. Z. 186 (1984), 407-417.

[BG] Yu. Brudny i and M. Ganzburg, On an extremal problem for polynomials of $n$ variables, Math. USSR Izv. 37 (1973), 344-356.

[BLMT] L. Bos, N. Levenberg, P. Milman, and B. A. Taylor, Tangential Markov inequalities characterize algebraic submanifolds of $\mathbb{R}^{n}$, Indiana Univ. Math. J. 44 (1995), 115138.

[BM] L. Bos and P. Milman, Sobolev-Gagliardo-Nirenberg and Markov type inequalities on subanalytic domains, Geometric and Func. Analysis 5 (1995), 853-923.

[Br] A. Brudnyi, A Bernstein-type inequality for algebraic functions, Indiana Univ. Math. J. 46 (1997), 93-116.

[FN1] C. Fefferman and R. Narasimhan, On the polynomial-like behavior of certain algebraic functions, Ann. Inst. Fourier 44 (1994), 1091-1179.

[FN2] A local Bernstein inequality on real algebraic varieties, Math. Z. 223 (1996), 673-692.

[FN3] Bernstein's inequality and the resolution of spaces of analytic functions, Duke Math. J. 81 (1995), 77-98 (1996).

[H] W. K. Hayman, Differential inequalities and local valency, Pacific J. of Math. 44 (1973), 117-137.

[G] G. M. Goluzin, Geometric theory of functions of a complex variable (English transl.), Transl. of Math. Monographs, Vol. 26, A.M.S., Providence, RI, 1969.

[K] M. KLimek, Pluripotential Theory, London Math. Soc. Monographs 6, Oxford Univ. Press, 1991.

[LL] B. Ya. Levin and V. N. Logvinenko, Classes of functions that are subharmonic in $\mathbb{R}^{m}$ and bounded on certain sets, in Investigations in Linear Operators and Function Theory 17, 157-175, Zapiski Nauchnyh Seminarov LOMI, "Nauka" Leningrad, 1989.

[P] B. P. Panejah, Certain inequalities for functions of exponential type and a priori estimates for general differential operators, Russian Math. Surveys 21 (1966), 75114.

[R] E. Remez, Sur une propiérté extrémale des polynômes de Tchebychef, Zap. NaukDoslid. Inst. Mat. Meh., Harkiv, Mat. Tovar. 13 (1936), 93-95.

[RY] N. Roytvarf and Y. Yomdin, Bernstein classes, Ann. Inst. Fourier 47 (1997), 825858 .

[S] A. Sadullaev, An estimate for polynomials on analytical sets, Math. USSR Izv. 20 (1983), 493-502.

[St] E. M. Stein, Beijing lectures in harmonic analysis (ed.), Annals of Math. Studies 112, Princeton Univ. Press, Princeton, NJ, 305-307.

(Received May 23, 1997) 